Adzo DZIFA KoKUTSE ${ }^{1}$ KOSSI ADJONOU ${ }^{1}$ KOUAMI KOKOU ${ }^{1}$ MESSANVI GBeAsSOR ${ }^{2}$

1 Laboratoire de botanique et écologie végétale Faculté des Sciences Université de Lomé BP 1515, Lomé

Togo

${ }^{2}$ Laboratoire de pharmacologiephysiologie

des substances naturelles Faculté des Sciences Université de Lomé BP 1515, Lomé Togo

\section{Problématique \\ de la performance du teck
de provenance tanzanienne \\ de la performance du teck
de provenance tanzanienne par rapport au teck local
en plantation au Togo par rapport au teck local
en plantation au Togo}

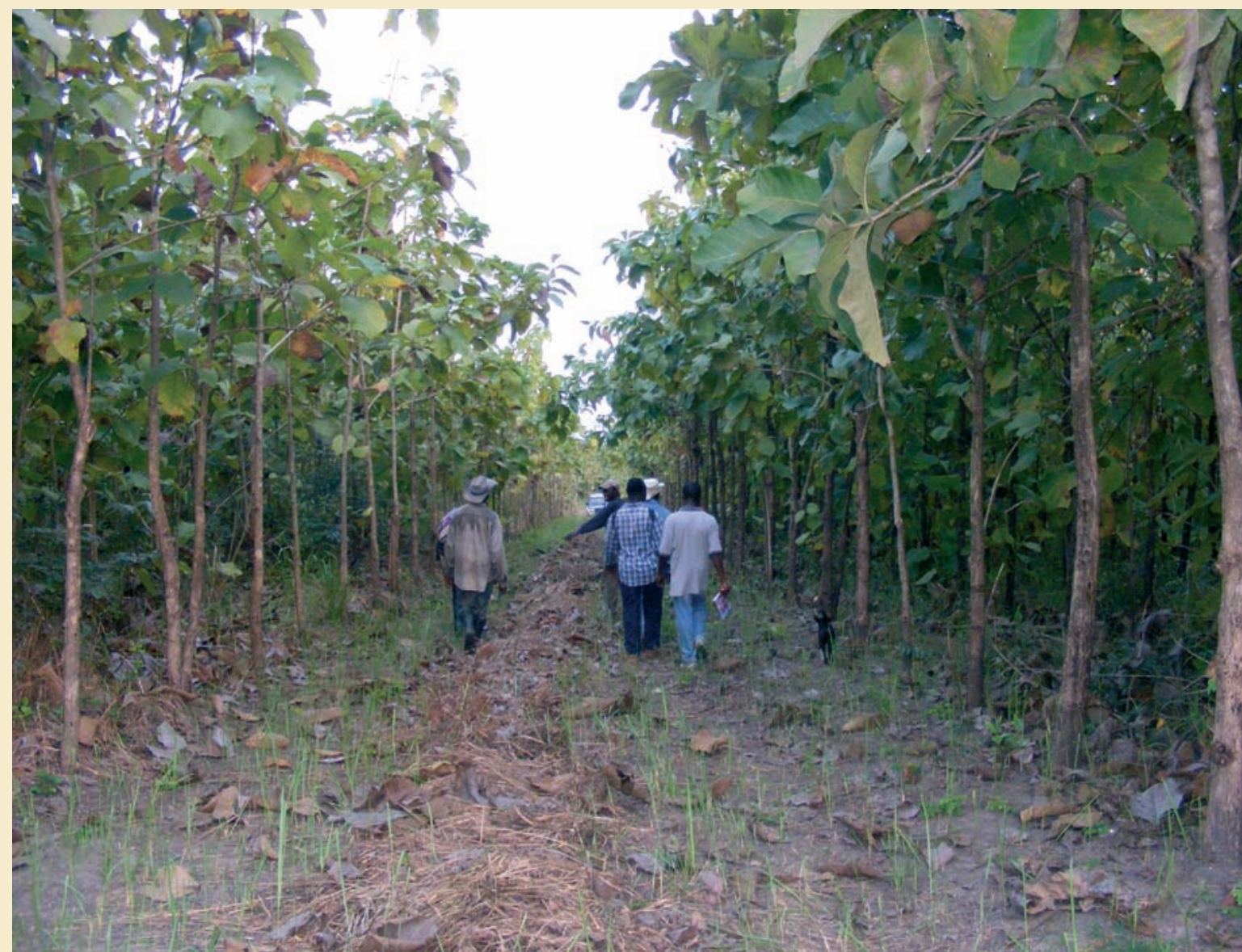

Photo 1.

Vue partielle de la forêt.

Photo A. D. Kokutse. 


\section{A. D. KoKUTSE, K. ADJONOU, K. KoKOU, M. GBEASSOR}

\section{RESUMEN}

\section{COMPARATIVE PERFORMANCE OF TANZANIAN TEAK VERSUS LOCAL TEAK PLANTED IN TOGO}

\section{PROBLÉMATIQUE \\ DE LA PERFORMANCE DU TECK DE PROVENANCE TANZANIENNE PAR RAPPORT AU TECK LOCAL EN PLANTATION AU TOGO}

Originaire d'Asie du Sud et introduit au Togo depuis le début du siècle dernier, le teck (Tectona grandis L.f.) y est devenu la première espèce commercialisable. Mais depuis les années 1990, une nouvelle provenance dite teck tanzanien, reconnue pour ses bonnes performances, est préférée par le service forestier et les planteurs privés. Cette étude compare les performances morphologiques et technologiques du teck tanzanien à celles $d u$ teck cultivé depuis un siècle au Togo. Pour ce faire, les caractéristiques dendrométriques, l'indice de productivité $\left(I_{p}\right)$, le pourcentage de bois de cœur à $1,30 \mathrm{~m}$ de hauteur de tige, les propriétés physiques et mécaniques ont été mesurés sur deux parcelles âgées de 7 ans. Les résultats montrent qu'à ce stade de croissance la provenance tanzanienne est plus productive $\left(I_{p}=6,75\right)$ que la provenance locale $\left(I_{p}\right.$ $=6,06$ ). Les hauteurs du teck tanzanien (hauteur dominante $\mathrm{H}_{0}$, hauteur totale $\mathrm{H}_{\mathrm{t}}$ et hauteur fût $\mathrm{H}_{\mathrm{f}}$ ) sont significativement supérieures à celles du teck local. En revanche, chez les arbres dominants, le teck local présente un diamètre moyen $D_{d}$ significativement supérieur à celui du teck tanzanien. La provenance tanzanienne renferme en moyenne $40 \%$ de plus de bois de cœur que le teck local. Cependant, les deux provenances ne montrent aucune différence significative en ce qui concerne les caractéristiques physiques (densité du bois à $12 \%$ d'humidité) et mécaniques du bois (module d'élasticité et contrainte de rupture en flexion).

Mots-clés : duramen, caractéristiques morphologiques, propriétés physiques et mécaniques, teck tanzanien.
Originated from South Asia and introduced in Togo at the beginning of the last century, the teak (Tectona grandis L.f.) became in this country the first marketable tree species. But since 1990, a new provenance of teak called "Tanzanian teak", known for its enhanced performances, is preferred by the forest service and the private planters. This survey compares morphological and technological performances of the tanzanian teak to those of the teak planted since one century in Togo. For instance, dendrometric characteristics, productivity index $\left(I_{\mathrm{p}}\right)$, percentage of heart wood at 1,30 m height of the stem, physical and mechanical properties have been measured in two parcels, old of 7 years. The results show that at this stage of growth, the Tanzanian provenance is more productive $\left(I_{p}=6,75\right)$ than the local provenance $\left(I_{p}=6,06\right)$. The height of the Tanzanian teak (dominating trees height $\mathrm{H}_{0}$, total height $\mathrm{H}_{t}$ and marketable height $\mathrm{H}_{\mathrm{f}}$ ) are significantly superior than those of the local teak. On the other hand, the local teak within the dominating trees presents a mean diameter $D_{d}$ significantly superior than the one of the Tanzanian teak. The tanzanian provenance contains average $40 \%$ more heart wood than the local teak. However, both provenances show no significant difference with regard to the physics (density of wood to $12 \%$ of humidity) and mechanical properties of the wood (modulus of elasticity and constraint of bending resistance).

Keywords: heart wood, morphological characteristics, physical and mechanical properties, tanzanian teak.

\section{PROBLEMÁTICA DEL DESEMPEÑO DE LA TECA DE ORIGEN TANZANO FRENTE A LA VARIEDAD LOCAL EN UNA PLANTACIÓN DE TOGO}

Originaria del sur de Asia e introducida en Togo a principios del siglo XX, la teca (Tectona grandis L.f.) se ha convertido en la primera especie comercializable. Pero, desde los años 90, los servicios forestales y los cultivadores privados prefieren una nueva procedencia, denominada teca de Tanzania, que destaca por su buen desempeño. Este estudio compara el desempeño morfológico y tecnológico de ambas especies de teca: la tanzana y la local. Para ello, se tomaron dos parcelas de 7 años de edad y se evaluaron las características dendrométricas, el índice de productividad $\left(I_{p}\right)$, el porcentaje de duramen a 1,30 m de altura del tronco y las propiedades físicas y mecánicas. Los resultados muestran que, en este estadio de crecimiento, la procedencia tanzana es más productiva $(I p=6,75)$ que la local $\left(I_{p}=6,06\right)$. Las alturas registradas en la teca de Tanzania (altura dominante $\mathrm{H}_{0}$, altura total $\mathrm{H}_{\mathrm{t}}$ y altura de fuste $\mathrm{H}_{\mathrm{f}}$ ) son significativamente superiores a las de la teca local. Sin embargo, en los árboles dominantes, la teca local presenta un diámetro medio $D_{d}$ significativamente superior al de la teca de Tanzania. La procedencia tanzana contiene, en promedio, un $40 \%$ más de duramen que la teca local. A pesar de ello, las dos procedencias no muestran ninguna diferencia significativa en cuanto a las características físicas (densidad de la madera al $12 \%$ de humedad) y mecánicas de la madera (módulo de elasticidad y tensión de ruptura en flexión).

Palabras clave: duramen, características morfológicas, propiedades físicas y mecánicas, teca de Tanzania. 


\section{Introduction}

Au Togo, les premières plantations de teck datent de l'époque coloniale. Les administrations allemande et française avaient mis en place des plantations et reboisements, essentiellement à base de teck (Tectona grandis), pour compenser la faible proportion de forêts naturelles (KOKUTSE, 2002 ; BEHAGHEL, 1999). En 1971, l'Odef ${ }^{1}$ fut créé pour gérer les plantations coloniales et mettre au point des techniques sylvicoles adaptées. C'est ainsi que, dans les années 1990, les superficies des plantations de teck de l'État ont atteint 14000 ha (Tengue, 1995). Puis les planteurs privés ont commencé à participer significativement à l'accroissement des superficies plantées en teck, principalement pour des besoins de bois de service et secondairement de bois d'œuvre. Ces initiatives font que les plantations forestières (toutes espèces confondues) avoisinent aujourd'hui 40000 ha, soit 1200 ha plantés par an (photo 1).

Dans les années 1990, un phénomène nouveau intervient dans cet engouement pour les plantations de teck. Il s'agit de l'introduction du teck dit de « provenance tanzanienne » ou «teck tanzanien». Bien que les premières semences proviennent de la Tanzanie, les graines actuellement vendues au Togo sont récoltées dans les teckeraies de seconde génération au Bénin, d'où le nom « provenance Bohicon » que lui préfèrent certains auteurs (ALI, 2002). Les informations sur les performances de ce teck ont été divulguées par l'Odef qui l'avait recommandé pour des plantations à grande échelle dans la forêt classée de HahoBaloé au sud du Togo, dans les années 1990. Il semble qu'il est d'une qualité supérieure au teck local du Togo : croissance rapide, bonne rectitude du tronc, hauteur plus grande et diamètre plus gros que le teck local, tige à ramification tardive, pas de floraison précoce, etc. Tous ces « atouts » ont fait grimper rapidement les surfaces plantées en teck au Togo. Il s'agirait d'un

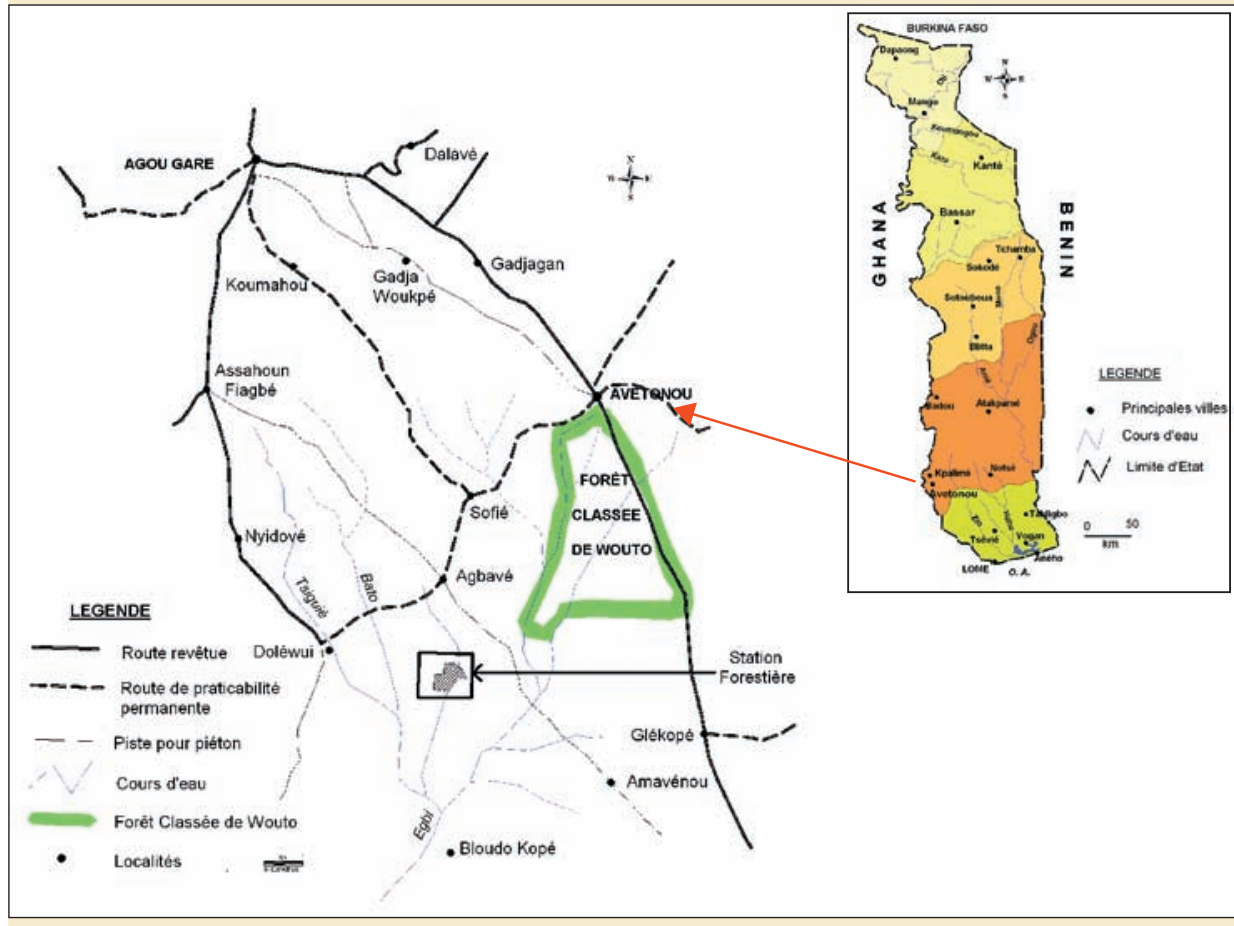

Figure 1.

Localisation de la station forestière.

phénomène régional car, dans les autres pays de la sous-région, les surfaces plantées en teck ont aussi augmenté : Bénin (15000 ha), Ghana (40 000 ha), Côte d'Ivoire (58 000 ha) et Nigeria (40 $000 \mathrm{ha}$ ) (Adu-Bredu et al., 2008). En conséquence, les graines ou jeunes plants du teck tanzanien sont devenus plus chers au Togo que le teck local (8 euros le kilogramme de graines de teck tanzanien contre 1 euro le kilogramme pour le teck local ; 20 centimes d'euro le plant tanzanien en pot contre 8 centimes d'euro le plant local en pot). Les pépiniéristes togolais vont souvent à Bohicon (Bénin) pour s'approvisionner en semences de teck tanzanien. Parallèlement, une filière illégale est née car certains pépiniéristes peu scrupuleux n'hésitent pas à vendre le teck local à la place du teck tanzanien.

Face à l'ampleur du phénomène, la Faculté des Sciences de l'Université de Lomé (Togo) a cherché à mieux comprendre le bien-fondé des performances tant louées chez le teck tanzanien. C'est ainsi que deux hectares de teck ont été mis en place en 2001, dans des conditions environnementales identiques (mêmes types de sol et de climat), dans une station forestière privée (un hectare de teck local et un hectare de teck tanzanien). Les deux parcelles ont subi des traitements sylvicoles identiques jusqu'à la première éclaircie en mars 2008. Plusieurs études sont en cours dans la station forestière d'Agbavé (voir ci-dessous), notamment la recherche sur l'appauvrissement de la végétation naturelle du sous-bois en fonction de la croissance des arbres, car le teck a la réputation d'éliminer naturellement le sous-bois, la variabilité morphologique, la modélisation de la croissance des arbres et l'étude de la duraminisation des arbres en fonction de l'âge et de la provenance. L'objectif de cet article est de comparer les caractères morphologiques des troncs, les caractères physiques et mécaniques du bois et la duraminisation des arbres au stade de perches avant les premières éclaircies survenues en mars 2008. 


\section{Matériels et méthodes}

\section{Cadre de l'étude}

La station forestière est localisée à Agbavé, un village situé à environ huit kilomètres d'Avétonou dans la préfecture d'Agou, au sud-ouest du Togo. Elle se trouve dans la pénéplaine granito-gneissique qui s'étend au pied du plateau de Kloto et du mont Agou jusqu'à la frontière du Ghana. Les sols sont ferrugineux, lessivés, indurés et parfois cuirassés. Le long des cours d'eau et dans certaines dépressions à inondation périodique, se rencontrent des sols constitués d'argiles montmorillonitiques. Le site est parcouru par des cours d'eau et des rivières de taille moyenne telles que Wouto, Tsiguié, Bato et Egbi (figure 1).
La zone bénéficie d'un climat de type guinéen caractérisé par quatre saisons. La pluviosité moyenne annuelle varie entre $1100 \mathrm{~mm}$ et $1400 \mathrm{~mm}$ avec un maximum au mois de juin. La température moyenne maximale varie entre $29^{\circ} \mathrm{C}$ et $36^{\circ} \mathrm{C}$. Les mois les plus chauds sont février et mars. Les moyennes minimales annuelles oscillent entre $20^{\circ} \mathrm{C}$ et $36^{\circ} \mathrm{C}$; les mois les moins chauds sont juillet et août. L'humidité relative de l'air se situe en moyenne autour de $83 \%$.

Le site d'implantation de la station forestière était soumis à une agriculture intensive, avec des périodes de jachère relativement courtes. Ce qui a entraîné l'appauvrissement des terres envahies par Imperata indica. Les cultures pratiquées par les populations locales sont le maïs, le manioc, le haricot, l'igname, l'arachide et le coton. Le palmier à huile est très présent dans le domaine. La

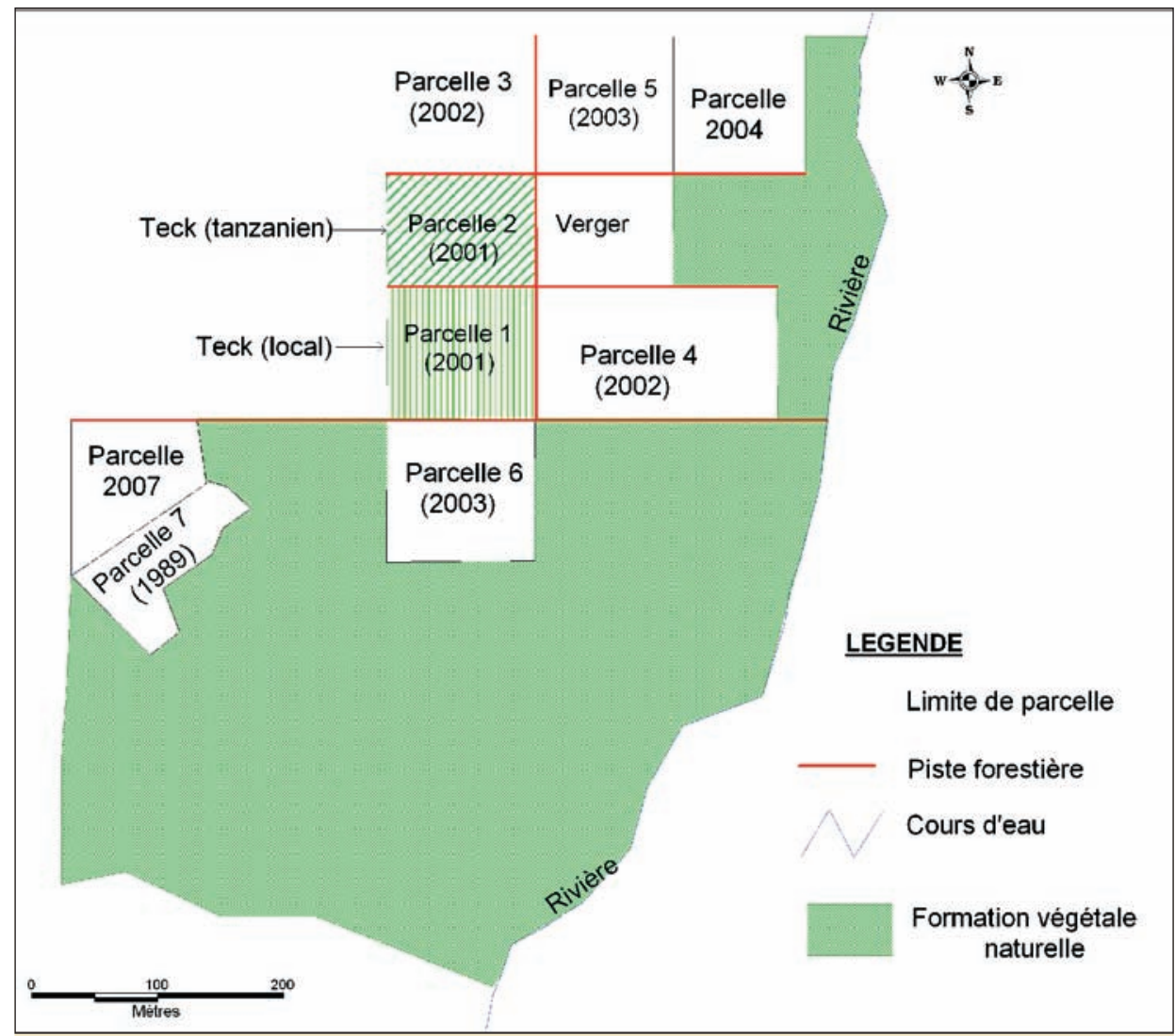

Figure 2

Localisation des parcelles 1 et 2 . végétation naturelle est la savane boisée guinéenne avec des forêts-galeries le long des cours d'eau. Ces savanes, reliques de galeries forestières et plantations de teck, offrent un gîte pour la faune sauvage devenue très rare dans la région.

La localité est peuplée par les Ewé venus vers la fin du XVle siècle de Tado. Cette peuplade autochtone majoritaire a vu, vers les années 1960, l'arrivée plus ou moins massive d'autres peuplades surtout venues de la partie septentrionale du pays. Ces immigrants (30\%) sont essentiellement constitués de Kabyè, Nawdeba, Kotocoli et Moba.

La station forestière couvre trente-sept hectares et a été achetée à des collectivités d'Agbavé (figure 1). Actuellement, 15 ha de teck d'âge compris entre 1 et 7 ans y sont plantés. L'étude concerne essentiellement les parcelles 1 (teck local) et 2 (teck provenance tanzanienne) (figure 2). Le teck tanzanien provient de plants en pots de la pépinière de l'Odef alors que la provenance locale est issue des teckeraies de l'Odef à Avétonou, connue comme la meilleure au Togo (photos 2 et 3). Il s'agit de monocultures de tecks plantés à intervalle de $2 \mathrm{~m} \times 2 \mathrm{~m}$, soit une densité initiale de 2500 pieds de teck par hectare.

\section{Historique de l'origine des premières graines}

Un certain doute existe sur l'origine des premières semences des tecks de Tanzanie. En fait, les tecks de Tanzanie auraient été introduits à partir de graines provenant du Myanmar (ex-Birmanie) ou de Java (Indonésie) (KEIDING, 1989). D'autres auteurs évoquent plutôt une origine indienne de ces graines (MAdoffe, MAGHEMBE, 1988). De la Tanzanie, les semences ont été très récemment introduites au Bénin, puis au Togo dans les années 1990 (ALI, 2002). En ce qui concerne le teck local du Togo, les premières plantations ont été réalisées en 1905 grâce à des graines qui seraient venues du Nigeria (SouvAnNAVONG, 1986). Les toutes premières planta- 
tions en Afrique de l'Ouest ont commencé au Nigeria en 1902 avec des semences provenant d'abord de l'Inde et de Myanmar (PANDEY, Brown, 2000 ; ALI, 2002). Grâce aux graines venues du Nigeria, le teck aurait été introduit plus tard au Bénin en 1916 par des missionnaires catholiques (Demenois, HeurtauX, 2001), au Togo (SouvanNavong, 1986) et ensuite en Côte d'Ivoire, en 1927, avec des graines provenant du Togo. Des études récentes ont été entreprises pour déterminer l'origine exacte des diverses populations de teck en plantation au Togo depuis un siècle (LOGOSSA, 2006).

\section{Méthode de l'étude}

\section{Mesure des caractéristiques dendrométriques}

Dans les parcelles 1 et 2 (figure 1), cent cinquante arbres ont été choisis au hasard. La hauteur totale $\left(H_{t}\right)$, la hauteur du fût $\left(H_{f}\right)$ et le diamètre à $1,30 \mathrm{~m}$ du sol ont été mesurés sur chacun des arbres. La surface terrière $(\mathrm{G})$ a été déterminée par la formule suivante :

$$
G=\sum \pi x D^{2} / 4
$$

(avec $\mathrm{G}$ en $\mathrm{m}^{2} /$ ha et $\mathrm{D}$ le diamètre des arbres).

La fonction allométrique exprimant la relation existant entre le diamètre à hauteur de poitrine $(1,30 \mathrm{~m}$ du sol) et la hauteur totale ou la hauteur fût est un indicateur de croissance des arbres. Elle a été exprimée par une régression logarithmique d'équation $Y=a x \operatorname{Ln}(X)+b(Y=$ hauteur, $X=$ diamètre à 1,30 du sol, a et b sont des constantes). La comparaison des caractéristiques dendrométriques entre les provenances tanzanienne et locale a été réalisée par analyse de variance grâce au programme Minitab.

L'indice de productivité $\left(\mathrm{I}_{\mathrm{p}}\right)$ traduit les performances dendrométriques, notamment la hauteur dominante vis-àvis de la fertilité des parcelles. Il a été calculé sur la base de la table de pro-

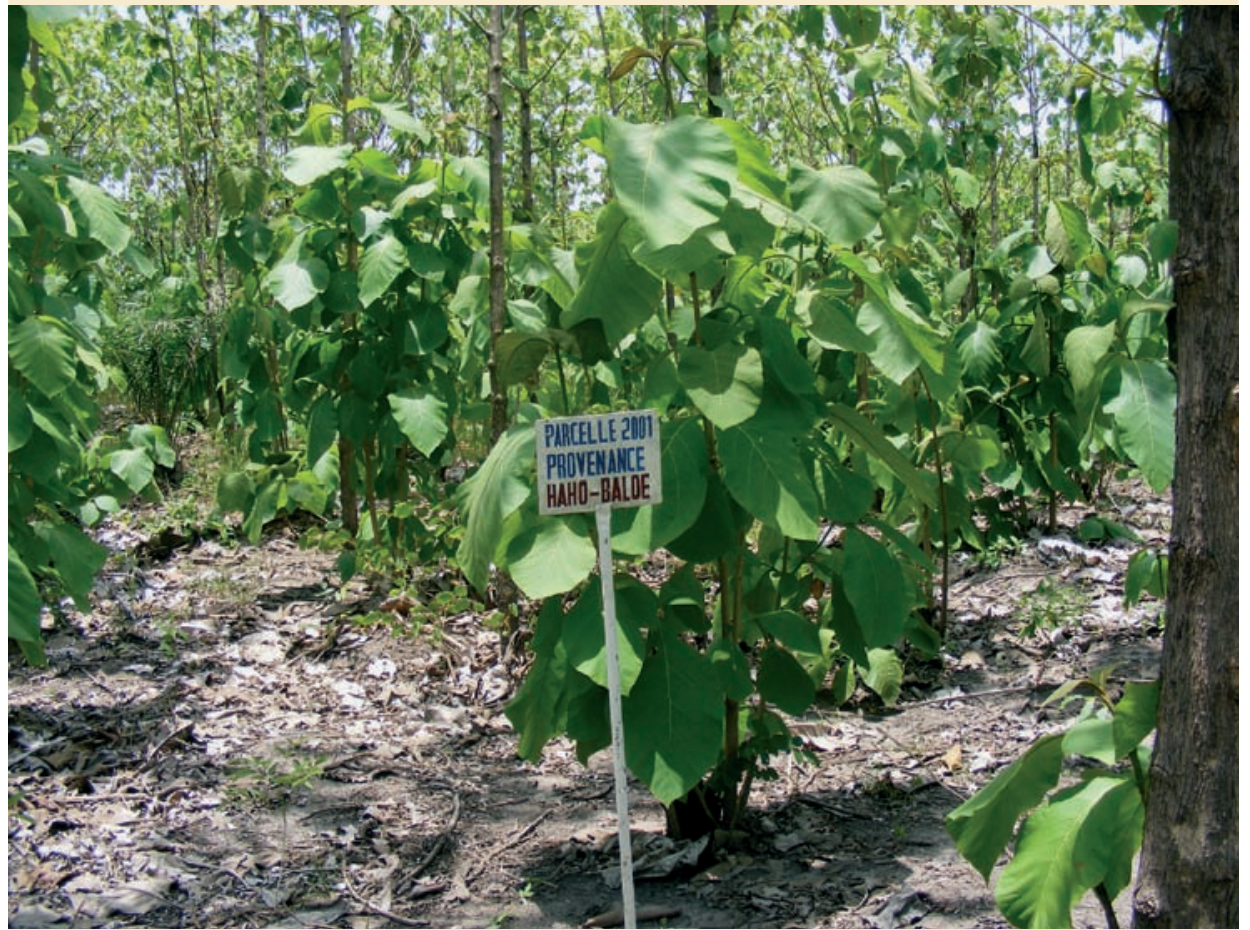

Photo 2.

Parcelle de teck tanzanien issu de pépinière réalisée à Haho-Baloe.

Photo A. D. Kokutse.

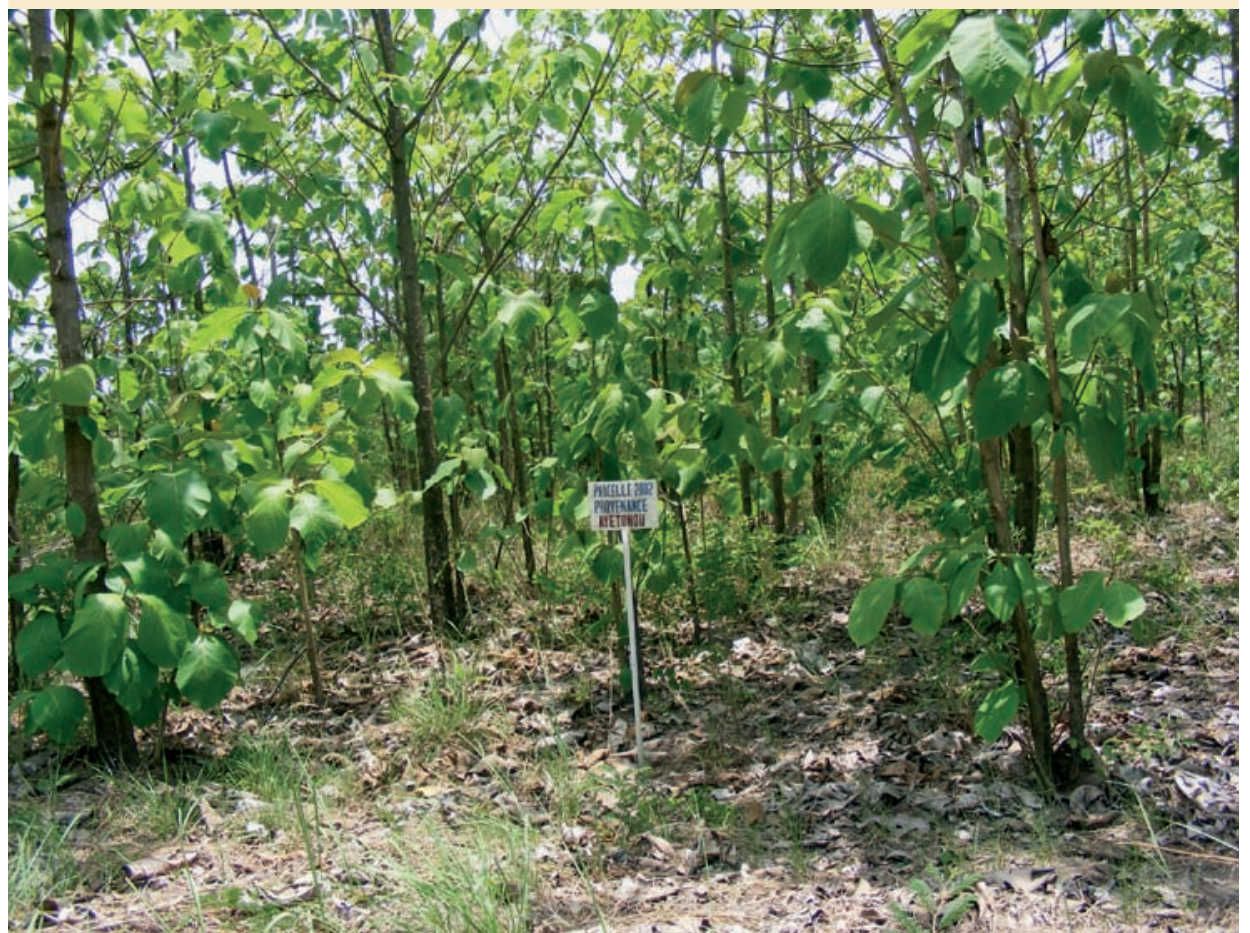

Photo 3.

Parcelle de teck local.

Photo A. D. Kokutse. 
ductivité de MAîTRE (1983), établie pour les peuplements de teck de la Côte d'Ivoire et adaptée aux teckeraies du Togo (TEngue, 1991) :

$$
\text { Ip }=H_{0} x\left(T^{0,304} x e^{0,910 / T}\right)
$$

avec $\mathrm{H}_{0}$ qui est la hauteur dominante (en mètres) et T l'âge du peuplement (en années).

\section{Mesure du pourcentage de bois de cœur}

Sur chacune des deux parcelles, dix arbres choisis au hasard ont été abattus. Deux rondelles d'environ $5 \mathrm{~cm}$ d'épaisseur ont été prélevées, une à la base et une à 1,30 m de hauteur de chaque tronc. Les surfaces supérieures des rondelles ont été rabotées, puis les rayons de la moelle à l'écorce et de la moelle à la limite du bois de cœur ont été mesurés (KoKUTSE et al., 2004). Le pourcentage de bois de cœur a été calculé par rapport à la surface totale de la rondelle (photo 4). Des analyses de variance ont été réalisées pour déterminer la variation du taux de bois de cœur formé en fonction de la provenance du bois.

\section{Mesure des caractéristiques physiques et mécaniques}

Mesure de la densité à $12 \%$ d'humidité. Une planchette de $50 \mathrm{~cm}$ de longueur et de $5 \mathrm{~cm}$ d'épaisseur a été prélevée radialement sur chaque arbre à 1,30 $\mathrm{m}$ de hauteur du tronc. Les planchettes ont été stabilisées à $12 \%$ d'humidité. Elles ont ensuite été débitées en éprouvettes non normalisées de $50 \mathrm{~cm} \times 1,5 \mathrm{~cm} \times 2 \mathrm{~cm}$ selon les directions longitudinale, tangentielle et radiale $(L, T, R)$. Les mesures de densité du bois ont été réalisées sur chaque éprouvette à l'aide d'une balance de pesée et d'un pied à coulisse servant à mesurer les cotes (hauteur et largeur) de l'éprouvette en trois points de sa longueur. La formule suivante a été utilisée pour calculer la densité de chaque éprouvette :

$\mathrm{D}=\mathrm{M} / \mathrm{V}$, avec $\mathrm{V}=\mathrm{L} \times \mathrm{I} \times \mathrm{e}, \mathrm{M}(\mathrm{g})$ = masse de l'éprouvette à $12 \%$ d'humidité, $\mathrm{L}(\mathrm{cm})=$ longueur de l'éprouvette à $12 \%$ d'humidité, l $(\mathrm{cm})=$ largeur moyenne de l'éprouvette à $12 \%$ d'humidité, e $(\mathrm{cm})=$ épaisseur moyenne de l'éprouvette à $12 \%$ d'humidité.

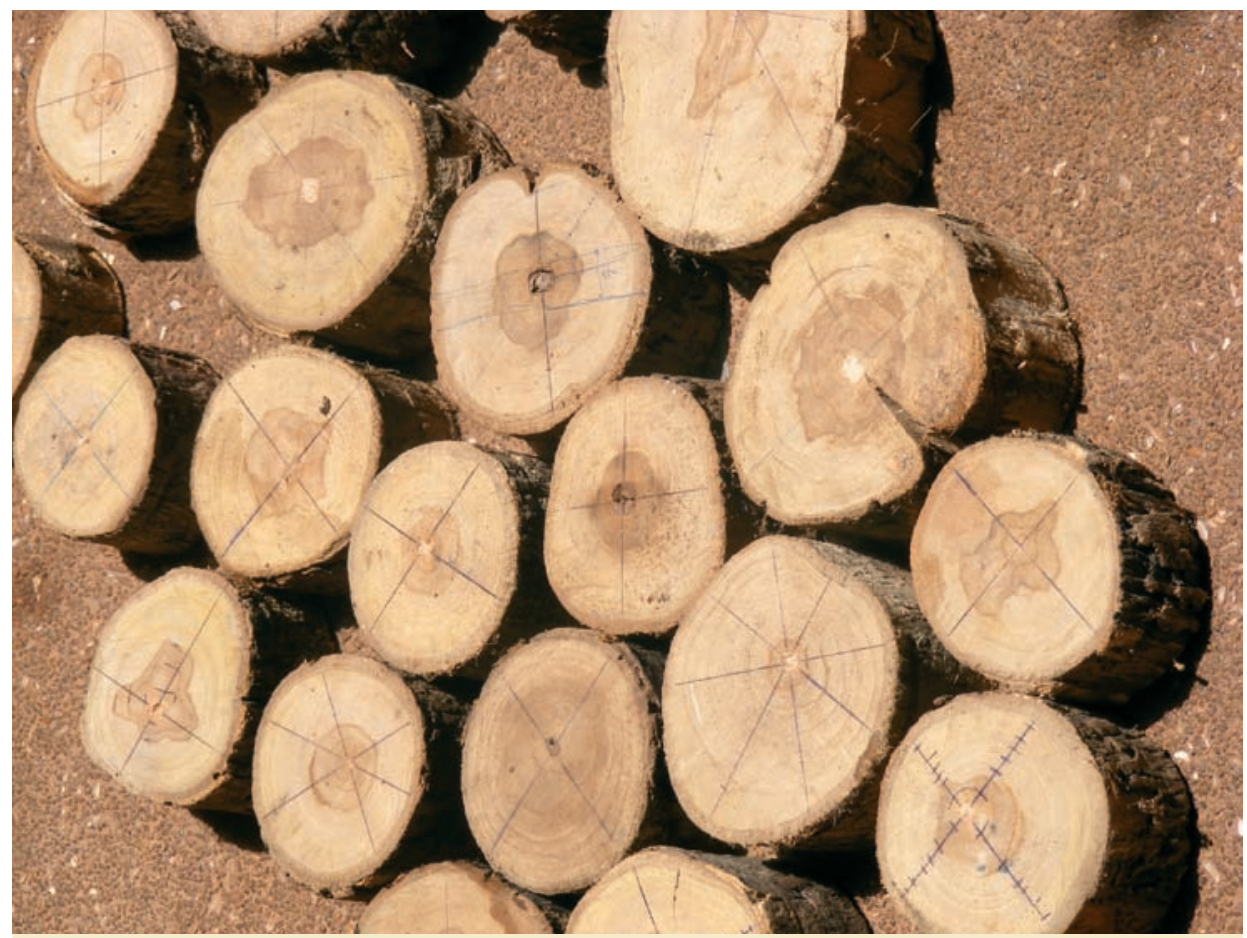

Photo 4.

Rondelles de teck préparées pour les mesures de bois de cœur. Photo A. D. Kokutse.
Mesure du module d'élasticité (EL). Les propriétés mécaniques (module d'élasticité et contrainte de rupture en flexion) ont été mesurées sur les mêmes éprouvettes que celles ayant servi à mesurer la densité du bois. La méthode utilisée est celle mise au point par le Cirad (BAILLÈres et al., 1998). Le principe de la mesure repose sur l'analyse spectrale des vibrations naturelles de flexion. Ce procédé permet de déterminer les fréquences propres d'une poutre à partir de sa réponse à une excitation appliquée à l'une des extrémités, de façon à solliciter simultanément tous les modes propres de vibration. La réponse est enregistrée à l'autre extrémité au moyen d'un microphone. Les vibrations sont ensuite transmises à un ordinateur. Le signal est traité par une procédure Fft (transformée de Fourrier). Un ajustement sur les premiers modes, basé sur la théorie de Timoshenko sur les poutres flottantes, permet de déterminer le module d'élasticité (BAILLĖ̀Es et al., 1998).

Mesure de la contrainte de rupture en flexion. La méthode de mesure est celle décrite par la norme B51-008 (1987). Les éprouvettes d'essai sont placées sur le banc de flexion de façon que la charge appliquée soit parallèle aux cernes du bois. La contrainte de rupture en flexion statique de l'éprouvette $(\sigma)$ est calculée par la formule :

$$
\sigma=\frac{3 P(l a)}{2 b h^{2}}
$$

avec : P la charge totale appliquée à la rupture, $l$ et a respectivement les distances entre les appuis et entre les axes de tête chargement, $b$ et $h$ respectivement la largeur et la hauteur de l'éprouvette. Des analyses de variance ont été réalisées pour déterminer la variation des caractéristiques physiques et mécaniques en fonction de la provenance du teck. 
Tableau I.

Caractéristiques dendrométriques des parcelles.

\begin{tabular}{|c|c|c|c|c|c|c|}
\hline Provenance & $\mathrm{D}_{\mathrm{m}}(\mathrm{cm})$ & $\mathrm{D}_{\mathrm{d}}(\mathrm{cm})$ & $\mathrm{H}_{\mathrm{t}}(\mathrm{m})$ & $\mathrm{H}_{0}(\mathrm{~m})$ & $\mathrm{H}_{\mathrm{f}}(\mathrm{m})$ & $\mathrm{G}\left(\mathrm{m}^{2} / \mathrm{ha}\right)$ \\
\hline Tanzanienne & $8,75 \pm 2,21$ & $10,02 \pm 1,49$ & $8,99 \pm 2,46$ & $10,03 \pm 1,96$ & $6,32 \pm 2,06$ & 15,97 \\
\hline Locale (Togo) & $9,09 \pm 2,49$ & $10,62 \pm 1,67$ & $7,95 \pm 2,07$ & $9,01 \pm 1,48$ & $4,37 \pm 1,59$ & 17,41 \\
\hline
\end{tabular}

$\mathrm{Dm}$ : diamètre moyen ; $\mathrm{Dd}$ : diamètre dominant ; $\mathrm{Ht}$ : hauteur totale moyenne ; $\mathrm{H}_{0}$ : hauteur dominante ; $H f$ : hauteur fût ; $G$ : surface terrière.

\section{Résultats}

\section{Caractéristiques morphologiques et productivité des deux provenances de teck}

Les résultats montrent que la provenance tanzanienne et la provenance locale ne présentent aucune différence significative en ce qui concerne leur diamètre moyen $D_{m}(P=0,195)$. Lorsqu'on considère seulement les arbres dominants, le teck local présente un diamètre moyen $D_{d}$ significativement plus important que le teck tanzanien $\left(F_{1,199}=7,15 ; P=0,008\right)$. Mais les hauteurs du teck tanzanien, hauteur totale $\left(\mathrm{H}_{\mathrm{t}}\right)$ et hauteur du fût $\left(\mathrm{H}_{\mathrm{f}}\right)$, sont significativement supérieures à celles du teck local $\left(F_{1,300}=17,12 ; P<0,001\right.$ pour $H_{t}$ et $F_{1,300}=93,26 ; P<0,001$ pour $\left.\mathrm{H}_{\mathrm{f}}\right)$. La hauteur dominante $\left(\mathrm{H}_{0}\right)$ est aussi significativement plus importante chez le teck tanzanien que chez le teck local avec $F_{1,199}=17,17 ; P<0,001$ (tableau I). La surface terrière $(\mathrm{G})$ de la provenance locale est de $17,41 \mathrm{~m}^{2} / \mathrm{ha}$ (tableau I). Cette valeur est supérieure à celle de la provenance tanzanienne $\left(15,97 \mathrm{~m}^{2} / \mathrm{ha}\right)$. Les relations allométriques de croissance qui existent entre les hauteurs totales et les diamètres à hauteur de poitrine $(1,30 \mathrm{~m})$ s'ajustent par une fonction logarithmique dont les équations sont respectivement :

$\mathrm{YH}_{\text {ttanzanien }}=6,17 \operatorname{Ln}(\mathrm{x})-4,16$ avec $R^{2}=0,54 ; P=0,001$;

$\mathrm{YH}_{\text {flocal }}=5,18 \operatorname{Ln}(\mathrm{x})-3,25$ avec $R^{2}=0,60 ; P<0,001$.

Cette relation confirme la performance du teck tanzanien en termes de croissance. En effet, à diamètre égal, le teck tanzanien est plus haut que le teck local (figure 3).

\section{Productivité des deux provenances}

Au-delà des considérations structurales, la fertilité des parcelles joue un rôle primordial dans la croissance des arbres. Cette fertilité est exprimée par l'indice de productivité $\left(I_{p}\right)$ qui constitue l'un des indicateurs d'appréciation. Le teck tanzanien et le teck local présentent un $I_{p}$ qui varie de moyen (provenance locale) à acceptable (provenance tanzanienne) (tableau II). La provenance tanzanienne est plus productive $\left(I_{p}=6,75\right)$ que la provenance locale $\left(I_{p}=6,06\right)$.

\section{Proportion du bois de cœur et propriétés du bois}

Chez la provenance tanzanienne, le pourcentage de bois de cœur à 1,30 m du tronc varie de 10,32 à $35 \%$. À la base du tronc, il varie de 15,82 à 34,13\%. Chez le teck local, le minimum de bois de cœur formé est de $1,72 \%$ à $1,30 \mathrm{~m}$ de hauteur de tronc et le maximum est de $22,92 \%$. À la base du tronc, le taux de bois de cœur varie de 5,64 à 21,61 \%. La provenance tanzanienne renferme en moyenne $40 \%$ de plus de bois de cœur que le teck local (photo 5). Cependant, l'analyse de variance montre qu'il n'existe pas de diffé-

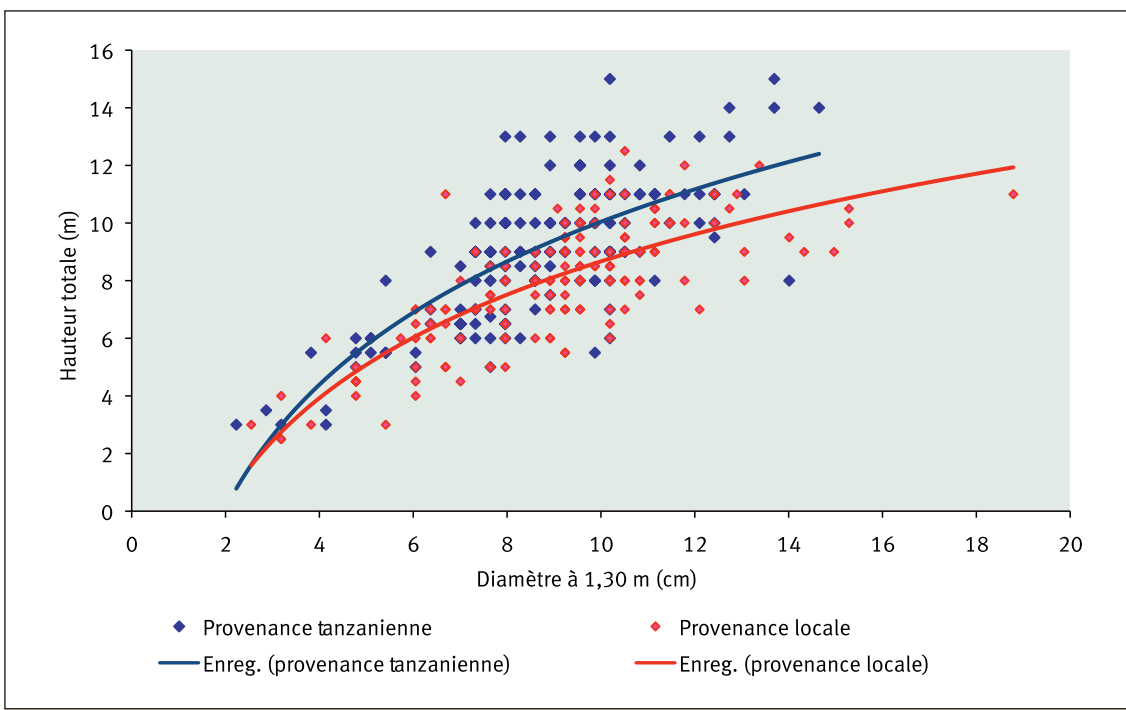

Figure 3.

Corrélation entre hauteur et diamètre à 1,30 m des arbres. 
Tableau II.

Comparaison de la productivité des deux parcelles.
Provenance

Origine tanzanienne

Origine locale
Hauteur dominante (m)

$9,01 \pm 1,48$
$10,03 \pm 1,96$
Indice de productivité

6,75

6,06 rence significative en ce qui concerne le taux de bois de cœur entre les deux provenances. Ce qui peut s'expliquer par la grande variabilité du pourcentage de bois de cœur entre les arbres à cet âge ; le coefficient de variation est d'environ $50 \%$ chez le teck tanzanien et $76 \%$ chez le teck local.

La densité du bois à $12 \%$ varie de 588 à 721 kg/m³ chez le teck tanzanien. Chez le teck local, le minimum est de $572 \mathrm{~kg} / \mathrm{m}^{3}$ et le maximum de densité est de $665 \mathrm{~kg} / \mathrm{m}^{3}$. La densité moyenne du bois de teck tanzanien est de $646 \pm 49 \mathrm{~kg} / \mathrm{m}^{3}$. Cette valeur est plus élevée que celle du teck local $\left(615 \pm 34 \mathrm{~kg} / \mathrm{m}^{3}\right)$. Toutefois, l'analyse de variance montre qu'il n'existe pas de différence significative entre les densités des deux provenances. Il faut noter que la variabilité de la densité du bois à ce stade est plus importante chez le teck tanzanien (coefficient de variation : $8 \%$ ) que chez le teck local (coefficient de variation : $5 \%$ ).

Le module d'élasticité $\left(E_{L}\right)$ varie respectivement de 10312 à $13557 \mathrm{MPa}$ et de 9168 à 12933 MPa chez la provenance tanzanienne et locale. Tout comme la densité, $E_{L}$ suit la même tendance. II est en moyenne plus élevé chez la provenance tanzanienne $(11885 \pm 1289 \mathrm{MPa})$ que chez le teck local (en moyenne $11447 \pm 1556 \mathrm{MPa}$ ). En ce qui concerne les contraintes de rupture, les valeurs sont comprises entre 68 et $88 \mathrm{MPa}$ chez la provenance tanzanienne et entre 69 et 86 MPa chez le teck local. L'analyse de variance ne montre cependant aucune différence significative entre les deux provenances de teck en ce qui concerne le module d'élasticité et la contrainte de rupture en flexion (tableau III).

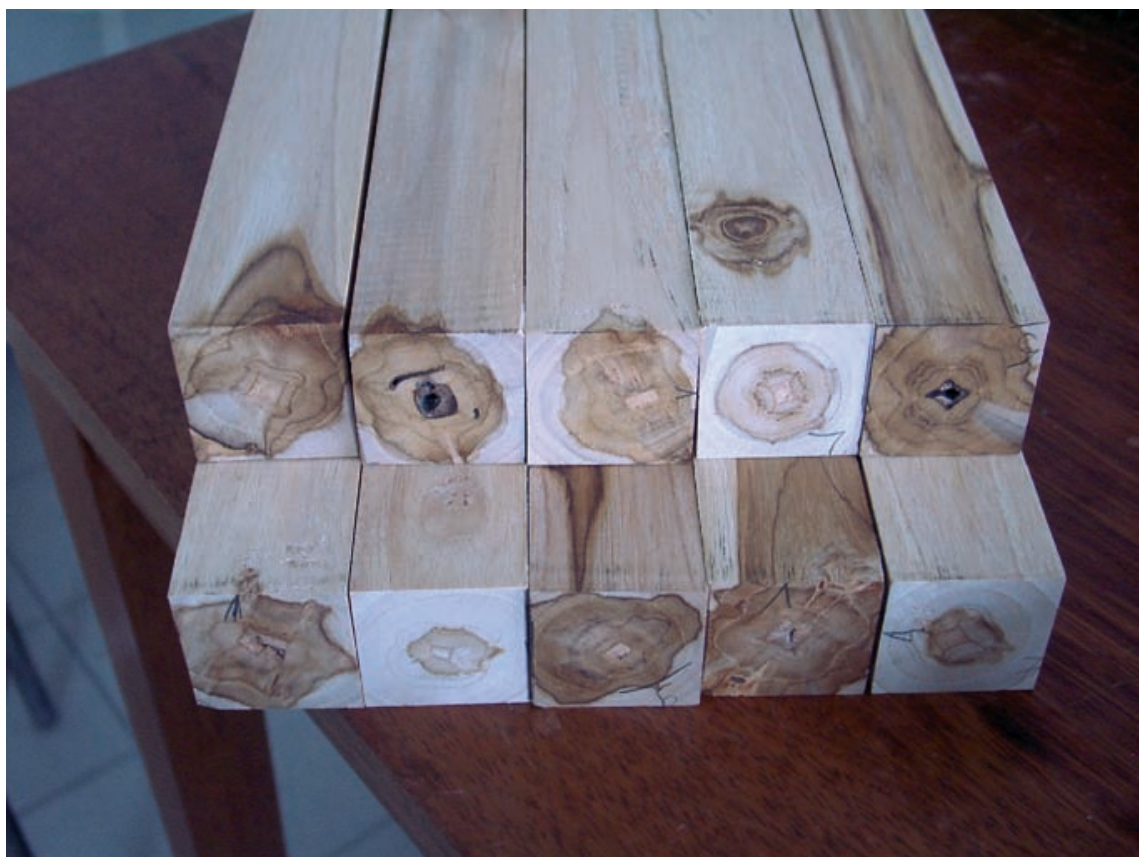

Photo 5.

Duramen dans les baguettes des deux provenances (les plus développés sont majoritairement le teck tanzanien).

Photo A. D. Kokutse.

\section{Discussion}

L'étude montre que les qualités qu'on prête au teck tanzanien sont justifiées ; les caractères morphologiques tels que la hauteur totale et la hauteur fût sont significativement plus importants chez le teck tanzanien. En revanche, la croissance en diamètre et la surface terrière sont plus élevées chez le teck local. Les deux provenances présentent des indices de productivité similaires et acceptables $\left(I_{p}>6\right)$ pour des plantations destinées à la production de bois d'œuvre (MAîTRE, 1983). Plusieurs études ont révélé des différences importantes entre les provenances de teck en termes de croissance et de caractéristiques externes du tronc (KEIDING et al., 1986 ; KJAER et al., 1995 ; PEDERSEN et al., 2007). Comparant les performances morphologiques de différentes provenances de teck, Pedersen et al. (2007) ont montré que l'origine affecte significativement le volume sur pied, la hauteur dominante et le diamètre dominant. KEIDING et al. (1986) ont aussi montré la bonne tenue du tronc des provenances tanzaniennes de teck grâce aux essais réalisés en Côte d'Ivoire et au Mexique. D'autres études encore ont montré des différences entre les provenances de teck (y compris les provenances de forêts naturelles) en termes de croissance et de rectitude des troncs (KEIDING et al., 1986 ; KumArAVELU, 1993 ; KJAER et al., 1995 ; KUANG et al., 1996). Mais il apparaît globalement que les différences significatives mises en évidence par ces différents travaux ne sont valides que pour les mêmes aires et sont difficiles à apprécier lorsqu'on ne travaille pas sur le même site.

Les mesures liées aux caractéristiques internes du bois montrent que la provenance du teck influence la formation du duramen. Bien que les tests statistiques ne soient pas significatifs, le pourcentage de bois de cœur est de $40 \%$ plus élevé chez le teck tanzanien. Des études réalisées sur le teck local âgé de douze ans dans la forêt classée de Wouto (figure 1) ont montré que le pourcentage de bois de cœur est en moyenne de $37 \%$ (KoKUTSE et al., 2004). Les 
Tableau III.

Pourcentage du bois de cœur et propriétés du bois des deux provenances.

Provenance

anzanienne

Locale
Pourcentage de bois de cœur à 1,30 m (\%)

$21,09 \pm 10,43$

$12,33 \pm 9,37$
Module

d’élasticité (MPa)

$11885 \pm 1289$

$11447 \pm 1556$
Densité

$\left(\mathrm{kg} / \mathrm{m}^{3}\right)$

$645,78 \pm 49,32$

$615,35 \pm 34,01$
Contrainte de rupture en flexion (MPa)

$81,18 \pm 7,68$

$81,72 \pm 9,97$ résultats de la présente étude montrent que le maximum de bois de cœur formé chez les provenances tanzaniennes de six ans est identique à celui trouvé chez le teck local de douze ans dans la même localité (KoKUTSE et al., 2004). La formation du bois de cœur est un caractère économique très important chez le teck ; le bois de cœur du teck est très durable et présente des qualités décoratives très attrayantes pour la fabrication des produits finis de qualité supérieure. De ce fait, les provenances de teck renfermant plus de bois de cœur sont les plus recherchées. D'autres études ont comparé le pourcentage de bois de cœur chez le teck en fonction des provenances. KJAER et al. (1999) ont montré que les provenances indiennes renferment plus de bois de cœur que les provenances indonésiennes et africaines. Le taux de bois de cœur important trouvé chez le teck tanzanien est un argument de plus pour son succès auprès des planteurs togolais.

Par ailleurs, cette étude montre qu'il n'existe aucune différence significative quant aux propriétés physiques et mécaniques entre le teck local et la provenance tanzanienne. La densité du teck local est en moyenne de $615 \mathrm{~kg} / \mathrm{m}^{3}$, ce qui confirme les résultats antérieurs dans la même localité, selon lesquels les arbres de la provenance locale de six ans présentaient des valeurs de densité variant entre 620 et $640 \mathrm{~kg} / \mathrm{m}^{3}$ (KoKUTSE et al., 2004). Peu d'études ont abordé les caractéristiques physiques et mécaniques du bois chez les tecks plantés en Afrique de l'Ouest (SANwo, 1987 ; BAILLÈreS, DURAND, 2000 ; KOKUTSE et al., 2004). En termes de densité et de module d'élasticité, les tecks du Togo, du Bénin et de la Côte d'Ivoire sont très proches (KoKUTSE et al., 2004). Une grande variabilité de ces caractères s'observe cependant entre arbres au sein d'une même parcelle, et entre peuplements (KOKUTSE, 2002 ; KOKUTSE et al., 2004). Au Togo, les études ont montré que ces variations observées sur le bois entre peuplements de teck tiennent probablement uniquement à des facteurs environnementaux (LogossA, 2006). À l'intérieur de chacune des deux provenances, la variabilité de densité révélée entre arbres par les résultats de la présente étude peut représenter des opportunités d'amélioration génétique au travers de la sélection des troncs présentant les meilleures aptitudes technologiques. Au Togo, cette variabilité observée à l'intérieur d'un même peuplement a certainement une corrélation avec les facteurs génétiques, d'autant plus que les effets génétiques au niveau individuel représentent $95 \%$ de tous les effets de la variabilité génétique étudiée au niveau des peuplements (LogOSSA, 2006).

Bien que n'étant probablement pas de même origine, le teck de provenance tanzanienne et le teck local du Togo présentent des caractères proches du point de vue technologique (propriétés physiques et mécaniques) mais sont plutôt différents en ce qui concerne les caractères morphologiques. Les variabilités morphologiques mises en évidence par la présente étude peuvent être effectivement liées à la provenance, si l'on considère que la provenance tanzanienne n'est pas originaire de l'Inde comme la provenance locale du Togo. II se pourrait aussi que les deux provenances soient originaires de l'Inde mais pas de la même localité en Inde. En effet, PEDERSEn et al. (2007) ont démontré que les caractères morphologiques pouvaient varier significativement entre individus provenant de différentes loca- lités d'une même région géographique (nord, centre ou est de l'Inde). Des études complémentaires s'avèrent de ce fait opportunes afin de déterminer l'origine exacte de ce teck tanzanien et de le situer par rapport aux tecks de forêts naturelles.

\section{Conclusion}

Au stade de perche, les caractères très prisés chez le teck tanzanien sont justifiés mais ne sont pas, pour la plupart, significatifs par rapport au teck local. Ils se limitent aux caractéristiques morphologiques (croissance en hauteur, indice de productivité, etc.) et à la proportion du bois de cœur dans les troncs. La provenance tanzanienne renferme en moyenne $40 \%$ de plus de bois de cœur, ce qui peut se révéler très important pour les producteurs si ce caractère se confirme chez les individus âgés (30-40 ans). Le teck local est plus performant à travers d'autres caractéristiques morphologiques telles que la croissance en diamètre et la productivité. Des caractères technologiques très importants chez le teck tels que la densité du bois et les propriétés mécaniques ne présentent pas, à cet âge, de différences significatives. Mais est-ce que ces caractères performants pourront persister chez les arbres au-delà du stade de perche et chez des générations futures de la même provenance ? Les échanges de gènes entre les provenances locales et tanzaniennes conduiraient-ils à long terme à un teck hybride qui cumule tous les caractères ? Les travaux futurs dans la station forestière apporteront des réponses à ces questions. 


\section{Références bibliographiques}

AFNOR, 1987. Norme NF B 51-008. Détermination de la résistance à la flexion statique de petites éprouvettes sans défaut. La Plaine SaintDenis, France, Afnor.

ADU-BREDU S., TAPE BI A. F., BOUILLET J. P., MÉ K. M., KYEI Y. S., SAINT-ANDRÉ J. P., 2008. An explicit stem profile model for forked and unforked teak (Tectona grandis) trees in West Africa. Forest Ecology and Management, 255 (7) : 2189-2203.

ALI S., 2002. Étude comparative de la croissance de deux provenances de teck (Tectona grandis L.f.) dans le système agrosylvicole Taugya à HahoBaloé (Togo). Mémoire de l’École d'ingénieurs en agronomie, Université de Lomé, Togo, 68 p.

BAILLÈRES H., DURAND P.-Y., 2000. Non destructive techniques for wood quality assessment of plantationgrown teak. Bois et Forêts des Tropiques, 263 (1) : 17-28.

BAILLÈRES H., CALCHERA G., DEMAY L., VERNAY M., 1998. Mechanical grading of French Guianan structural timber using three non-destructive techniques. Bois et Forêts des Tropiques, 257 : 54-64.

BEHAGHEL I., 1999. État des plantations de teck (Tectona grandis L.f.) dans le monde. Bois et Forêts des Tropiques, $262:$ 5-18

DEMENOIS J., HEURTAUX A., 2001. La filière teck en Inde du Sud (Karnataka, Kérala, Tamil Nadu). De la plantation à la commercialisation du teck : aspects sociaux, économiques et techniques. Mémoire de l'Engref/Cirad-Forêt/Institut français de Pondichéry, 117 p.

KEIDING H., 1989. Seed collection from teak plantations at Mtibwa and Longuza, Tanzania. Humlebæk, Danemark, Danida Forest Seed Centre, $7 \mathrm{p}$.
KJAER E. D., KAJORNSRICHON S., LAURIDSEN E. B., 1999. Heartwood, calcium and silica content in five provenances of Teak (Tectona grandis). Silvae Genetica, 48 : 1-3.

KJAER E. D., LAURIDSEN E. B., WELLENDORF H., 1995. Second evaluation of an international series of teak provenance trials. Humlebæk, Danemark, Danida Forest Seed Centre (Danish Centre for Forest, Landscape and Planning), 118 p.

KOKUTSE A. D., 2002. Analyse de la qualité du bois de teck (Tectona grandis L.f.) en plantation au Togo : formation du bois de cœur, propriétés mécaniques et durabilité. Thèse de doctorat, Université Bordeaux I, France, $142 \mathrm{p}$.

KOKUTSE A. D., BAILLÈRES H., STOKES A., KOKOU K., 2004. Proportion and quality of heartwood in Togolese teak (Tectona grandis L.f.). Forest Ecology and Management, 189 : 37-48.

KUANG B., ZHEN S., LUO M., LIN M., 1996. Evaluation of aggregate genetic value of main characters of provenances of teak. Forest Research, $9: 7-14$

KUMAREVELU G., 1993. Teak in India. In : Wood H. (éd.). Teak in Asia. Bangkok, Thaïlande, Forspa Publication, 4, 27-34.

LOGOSSA Z. T., 2006. Caractérisation génétique des Tecks (Tectona grandis L.f.) provenant de plantations du Togo. Mémoire de master II, Sciences du bois et fibres, Université Bordeaux 1, France, 29 p.

MADOFFE S. S., MAGHEMBE J. A., 1988. Performance of teak (Tectona grandis) provenances seventeen years after planting at Longusa, Tanzania. Silvae Genetica, 37 : 175-178.

MAîTRE H.-F., 1983. Table de production provisoire du teck en Côte d'Ivoire. Abidjan, Côte d'Ivoire, Ctft, 75 p.
DUPUY B., MAÎTRE H.-F., N'GUESSAN A. K., 1999. Table de production du teck (Tectona grandis), l'exemple de la Côte d'Ivoire. Bois et Forêts des Topiques, 261 (3) : 6-16.

PANDEY D., BROWN C., 2000. Le teck dans le monde. Unasylva, 201 : 3-13. PERDERSEN A. P., HANSEN K. J., MTIKA J. M., MSANGI T. H., 2007. Growth, stem quality and age-age correlations in a teak provenance trial in Tanzania. Silvae Genetica, 56 : 142-148.

SANWO S. K., 1987. The characteristics of the crown-formed and stemformed wood in plantation grown teak (Tectona grandis L.f.) in Nigeria. Journal of the Institute of Wood Science, $11: 85-88$.

SOUVANNAVONG O., 1986. Les teckeraies togolaises. Aménagement des parcelles à objectif bois d'œuvre. Lomé, Togo, Odef, 296 p.

TENGUE K. T., 1991. Tarifs de cubage du teck en plantation au Togo. Lomé, Togo, Odef, $13 \mathrm{p}$.

TENGUE K. M., 1995. Examen mi-parcours de 1995, rapport interne de l'Office de développement et d'exploitation des forêts du Togo «l'objectif an 2000 de l'OIBT ». Lomé, Togo, Odef, 10 p. 